\title{
EMBEDDING NUMBERS FOR FINITE GROUPS
}

\author{
JOHN ERNEST ${ }^{1}$
}

This note is concerned with the following problem. Let $H$ denote a subgroup of a finite group $G$ and let $L$ denote a linear or one dimensional representation (i.e., a character) of $H$. We assume throughout that the field $F$ is algebraically closed and is either of characteristic 0 or of prime characteristic which does not divide the order of any groups under consideration. Let $G \mid L$ denote the corresponding induced representation of $G$. How many distinct (i.e., nonequivalent) irreducible representations appear in the decomposition of $G \mid L$ into irreducible parts? (This number is just the central intertwining number of $G \mid L$, which is denoted by $\operatorname{eg}(G \mid L)$. Cf. [1].) More specifically, we are interested in determining an upper bound on the number of distinct irreducible representations which will appear, purely in terms of the way $H$ is embedded in $G$, and in terms which do not depend on the particular linear representation $L$ of $H$. Two such bounds come quickly to mind. The number of classes (of conjugates) of the super group $G$, which we denote $\{G: e\}$, is clearly an upper bound. Dimension considerations also give $[G: H]$ as an upper bound. We now introduce a new group theoretic invariant which heuristically is a measure of the manner in which the classes of $G$ are distributed among the $H$-cosets of $G$.

Definition. Let $H$ be a (not necessarily normal) subgroup of a finite group $G$. For each normal subset $N$ of $G$, let $\phi_{1}(N)$ denote the number of classes (of conjugates) of $G$ contained in $N$. Let $\phi_{2}(N)$ denote the number of right $H$-cosets of $G$ which have nonzero intersection with $N$. Let $\phi(N)=\{G: e\}-\phi_{1}(N)+\phi_{2}(N)$. We then define the embedding number of $H$ in $G$, denoted by $(G: H)$, to be the minimum of the $\phi(N)$, as $N$ is taken over all normal subsets of $G$. We remark that a definition of $\phi_{2}$ using left cosets would yield the same value for $(G: H)$ since $N^{-1}$ intersects the same number of left cosets as $N$ does right cosets.

Taking $N=\{e\}$ where $e$ is the identity element of the group we have $(G: H) \leqq\{G: e\}$. Taking $N=G$ we have $(G: H) \leqq[G: H]$. If $H \neq G$, it is easy to verify that $(G: H)>1$. If $H$ is a proper normal subgroup, then, taking $N=H$ we have $(G: H)<\{G: e\}$. In the case where $H$ is a normal subgroup of $G$, another number associated with the embedding of $H$ in $G$ is the number of classes in the factor group $G / H$. We call this the class number of $H$ in $G$ and denote it by $\{G: H\}$.

Received by the editors May 31, 1961.

1 National Science Foundation Fellow. 
Proposition 1. If $H$ is a normal subgroup of a finite group $G$, then $\{G: H\} \leqq(G: H)$.

Proof. Suppose $(G: H)=m$. Then there exists a normal subset, say $N$ of $G$, such that $N$ contains $n$ classes of $G$ and intersects $c H$-cosets such that $m=\{G: e\}-n+c$. Note that $n \geqq c$ since $m=(G: H)$ $\leqq\{G: e\}$. Let $\bar{N}$ denote the smallest normal subset of the factor group $G / H$, containing the $c H$-cosets which have nonzero intersection with $N$. Then the number of classes of $G / H$ contained in $\bar{N}$ is less than or equal to $c$. Let $N^{\prime}=\{x: x \in G$ and $x$ is contained in some $H$-coset belonging to $\bar{N}$. Then $N^{\prime}$ is a normal subset of $G$ such that $N \subset N^{\prime}$ and thus $N^{\prime}$ contains at least $n$ classes of $G$. Then $\left(G-N^{\prime}\right)$ is a normal subset of $G$ and contains at most $\{G: e\}-n$ classes of $G$. Thus $(G / H-\bar{N})$ contains at most $\{G: e\}-n$ classes of $G / H$. Further $\bar{N}$ contains at most $c$ classes. Hence $G / H$ contains at most $\{G: e\}-n+c$ $=m$ classes. Hence $\{G: H\} \leqq(G: H)$.

Thus in general we have $\{G: H\} \leqq(G: H) \leqq[G: H]$. If $G / H$ is abelian this degenerates to $\{G: H\}=(G: H)=[G: H]$. Now that we have a relative idea of how this new "embedding number" compares with the group theoretic invariants usually associated with the embedding of $H$ in $G$, we proceed to show the significance of $(G: H)$ in the theory of monomial representations. We must first prove a preliminary result.

Leмма. Let $H$ be a subgroup of a finite group $G$ and let $L$ denote $a$ linear representation of $H$, over the field $F$. Let $G \mid L$ denote the corresponding induced representation of $G$. Let $D_{1}, D_{2}, \cdots, D_{n+1}$ denote distinct classes of $G$ and let $S_{i}=\sum_{x \in D_{i}}(G \mid L)_{x}$, for $i=1,2, \cdots, n+1$. If these $n+1$ classes are completely contained in the union of $n$ right $H$ cosets of $G$, then the $S_{i}, i=1,2, \cdots, n+1$, are linearly dependent over F.

Proof. Index the right $H$-cosets of $G,\left\{H \sigma_{j}\right\}, j=1, \cdots, k$, in such a way that $D_{i} \subset \bigcup_{j=1}^{n} H \sigma_{j}$, for $i=1,2, \cdots, n+1$. Then $\left\{\sigma_{j}^{-1}: j=1, \cdots, k\right\}$ form a set of representatives of the left $H$-cosets of $G$. By [1, Corollary to Theorem 3], it is sufficient to show that there exists $\alpha_{1}, \alpha_{2}, \cdots, \alpha_{n+1}$ in $F$, not all zero, such that $\sum_{i=1}^{n+1} \alpha_{i} \beta_{i j}$ $=0$ for $j=1, \cdots, k$, where $\beta_{i j}=\sum_{x \in \sigma_{i}^{-1} D_{i} \cap H} L_{x}$ and $\beta_{i j}=0$ if $\sigma_{j}^{-1} D_{i}$ $\cap H$ is empty. Since $L$ is linear we have $\beta_{i j} \in F$. Consider the set of homogeneous linear equations

$$
\sum_{i=1}^{n+1} \beta_{i j} x_{i}=0, \quad j=1, \cdots, n .
$$

This system has $n$ equations and $n+1$ unknowns and thus has a non- 
trivial solution, say $x_{i}=\alpha_{i} \in F$. Hence $\sum_{i=1}^{n+1} \alpha_{i} \beta_{i j}=0$ for $j=1, \cdots, n$. By our indexing of the $H$-cosets we have that $\sigma_{j}^{-1} D_{i} \cap H=D_{i} \sigma_{j}^{-1} \cap H$ $=\left(D_{i} \cap H \sigma_{j}\right) \sigma_{j}^{-1}$ is empty (and thus $\beta_{i j}=0$ ), for $j>n$ and $i=1, \cdots$, $n+1$. Hence $\sum_{i=1}^{n+1} \alpha_{i} \beta_{i j}=0$ for $j=1, \cdots, k$.

TheOReM. Let $H$ denote a subgroup of a finite group $G$ and let $L$ be a linear representation of $H$. Then the number of distinct irreducible representations appearing in the decomposition of the induced representation $G \mid L$ is less than or equal to $(G: H)$.

Proof. There exists a normal subset $N$ of $G$ such that $(G: H)$ $=n-m+\phi_{2}(N)$, where $n=\{G: e\}$ and $m=\phi_{1}(N)$. Let $C_{1}, C_{2}, \cdots, C_{m}$ denote the classes of $G$ which are contained in $N$ and let $C_{m+1}$, $C_{m+2}, \cdots, C_{n}$ denote the remaining classes of $G$. Let $S_{i}$ $=\sum_{x \in C_{i}}(G \mid L)_{x}$, for $i=1,2, \cdots, n$. By the previous lemma there are at most $\phi_{2}(N)$ elements among the $S_{i}, i \leqq i \leqq m$, which are linearly independent over the field $F$. Hence there are at most $n-m+\phi_{2}(N)$ $=(G: H)$ linearly independent elements among the $S_{i}, 1 \leqq i \leqq n$. By [1, Theorem 1], $\operatorname{eg}(G \mid L) \leqq(G: H)$. That is to say, the number of distinct irreducible representations appearing in the decomposition of $G \mid L$ is less than or equal to $(G: H)$.

Corollary. Let $H$ denote an abelian subgroup of a finite group $G$. Then $\{G: e\} \leqq(G: H)[H: e]$.

Proof. Let $L$ denote the regular representation of $H$. Then $\mathfrak{C g}(L)=\{H: e\}=[H: e]$ and each irreducible representation appearing in the decomposition of $L$ is linear. Thus by the theorem $\operatorname{eg}(G \mid L)$ $\leqq(G: H)[H: e]$. But $G \mid L$ is the regular representation of $G$ and thus $\operatorname{eg}(G \mid L)=\{G: e\}$.

REMARK. ${ }^{2}$ If $H$ is a normal subgroup of $G$ and $L$ is the one-dimensional identity representation of $H$, then $G \mid L$ contains exactly $\{G: H\}$ distinct irreducible representations of $G$. Indeed it is sufficient to note that $G \mid L$ is the composition of the natural projection of $G$ on $G / H$ and the regular representation of $G / H$. The following proposition gives a sufficient condition for $\{G: H\}$ to be an upper bound to the number of distinct irreducible representations appearing in the decomposition of $G \mid L$, where $L$ is any linear representation of $H$. The referee conjectures that $\{G: H\}$ is such an upper bound whenever $H$ is a normal subgroup of $G$.

Proposition 2. Suppose $H$ is a normal subgroup of $G$ such that each class of $H$ is also a class of $G$. Then for every linear representation $L$ of $H, G \mid L$ contains at most $\{G: H\}$ distinct irreducible representations.

2 We are indebted to the referee for this remark. 
Proof. The projection of each class of $G$ onto $G / H$ is contained in a class of $G / H$. Suppose $D_{1}$ and $D_{2}$ are two classes of $G$ whose projections on $G / H$ are contained in the same class of $G / H$. Then the projections of $D_{1}$ and $D_{2}$ on $G / H$ have nonempty intersection. Thus there exists $x \in D_{1}, y \in D_{2}$ and $h \in H$ such that $x=h y$.

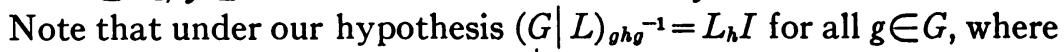
$I$ is the identity operator on $\operatorname{HC}(G \mid L)$. Indeed for all $g, z \in G$, and $f \in \mathfrak{H C}(G \mid L)$ we have $(G \mid L)_{o h \sigma^{-1}} f(z)=f\left(z g h g^{-1}\right)=L_{z g h \sigma^{-1}} z^{-1} f(z)=L_{h} f(z)$, where we have used the fact that $L$ is constant on the classes of $H$.

Let $n_{i}$ denote the number of elements in the class $D_{i}$, for $i=1,2$. Then we have

$$
\begin{aligned}
S_{1}=\sum_{z \in D_{1}}(G \mid L)_{z} & =\frac{n_{1}}{[G: e]} \sum_{o \in G}(G \mid L)_{o x o^{-1}} \\
& =\frac{n_{1}}{[G: e]} \sum_{o \in G}(G \mid L)_{o h o^{-1}(G \mid L)_{o y \sigma^{-}}} \\
& =\frac{n_{1}}{[G: e]} L_{h} \sum_{o \in G}(G \mid L)_{o y \sigma^{-}} \\
& =\frac{n_{1}}{n_{2}} L_{h} \sum_{z \in D_{2}}(G \mid L)_{z} \\
& =\left(\frac{n_{1}}{n_{2}} L_{h}\right) S_{2 .}
\end{aligned}
$$

Thus $\{G: H\}$ is an upper bound for the number of linearly independent conjugate sums $S_{i}$ and thus also for the number of distinct irreducible representations appearing in the decomposition of $(G \mid L)$.

For the theorem to have significance it is necessary to show that $(G: H)$ is indeed a better upper bound than those already known, namely $\{G: e\}$ and $[G: H]$. Let $G$ be the symmetric group on 4 letters. Let $H$ denote the normal abelian subgroup of $G$ of order 4 . Then all the numbers associated with the embedding of $H$ in $G$ are distinct. Indeed $[G: H]=6,\{G: e\}=5,(G: H)=4$ and $\{G: H\}=3$.

It would be interesting to know if the embedding number $(G: H)$ has any significance in any other context than in the theory of group representations which are induced from characters.

\section{REFERENCE}

1. J. A. Ernest, Central intertwining numbers for representations of finite groups, Trans. Amer. Math. Soc. 99 (1961), 499-508.

The Institute for Advanced Study and The UNIVERSITY OF ROCHESTER 